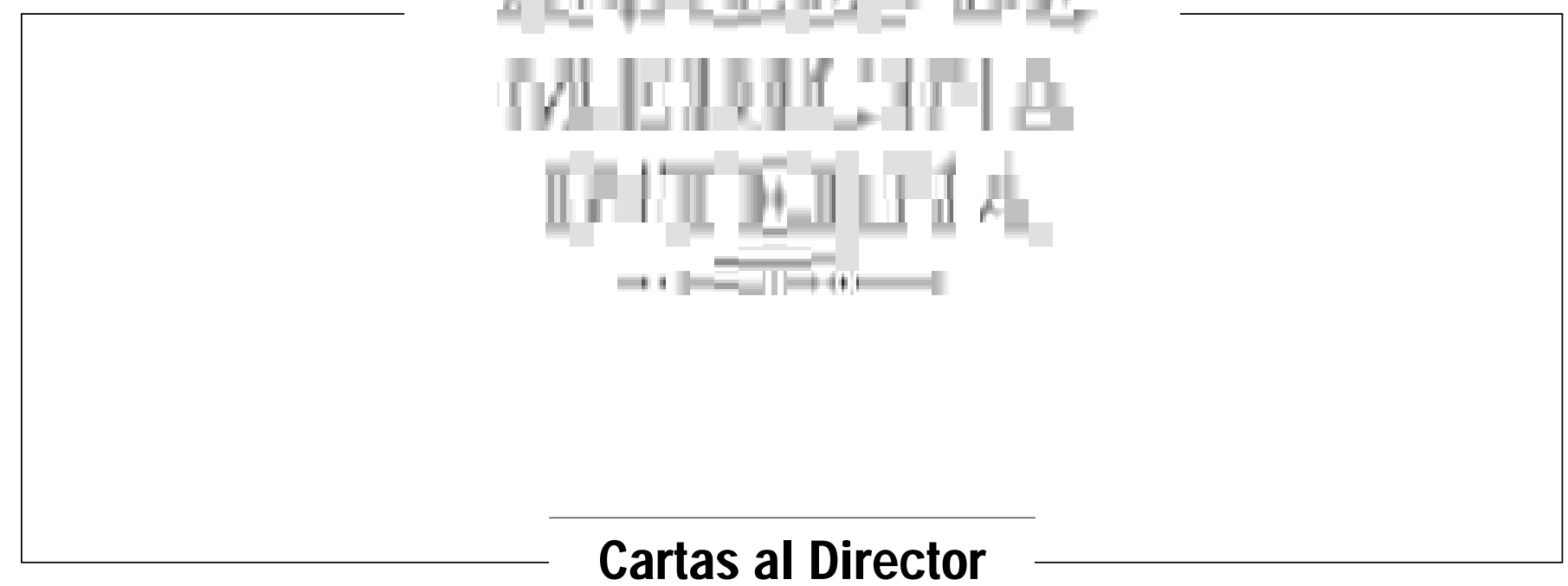

\section{Distrofia simpático refleja en el post-parto}

\section{Sr. Director:}

La distrofia simpático refleja (DSR) suele tener habitualmente un origen secundario, siendo el traumatismo la causa desencadenante más frecuente y, más excepcionalmente, el embarazo. Otras veces, no se encuentra una causa aparente, catalogándose como idiopática (1).

La DSR relacionada con el embarazo aparece, generalmente, en el último tercio del mismo. En menor medida, se ha descrito algún caso de DSR en el post-parto $(2,3)$. Teniendo en cuenta el carácter poco habitual de esta circunstancia, presentamos el caso de una DSR de la cadera izquierda acaecido a las tres semanas del parto.

Se trata de una mujer de 29 años que acude al Servicio de Urgencias por presentar, a las tres semanas de su segundo parto, dolor intenso en la parte anterior de la pierna izquierda, de aparición súbita, de tipo mecánico, que aumenta con el apoyo y con la marcha, acompañado de importante impotencia funcional, sin fiebre ni alteración del estado general. El cuadro clínico es catalogado, inicialmente, como una cruralgia y tratado con diclofenaco. No obstante, se solicita colaboración con el Servicio de Reumatología y se constata la misma sintomatología. En la exploración física se observa dificultad para la marcha y una movilización de la cadera izquierda dolorosa y parcialmente limitada, especialmente en sus rotaciones; sin evidenciarse signos externos de alteración vasomotora, ni afectación extensiva a otras articulaciones. Ante esta situación, se sospecha una DSR de la cadera y se solicitan exploraciones complementarias. En este sentido, la analítica no aporta parámetros de tipo inflamatorio, ni alteraciones metabólicas, ni sospecha de contexto infeccioso; la imagen radiológica de ambas caderas es idéntica y no presenta alteraciones; la gammagrafía ósea muestra una importante captación en el cótilo y en la cabeza femoral izquierda y en la RMN de la cadera izquierda se constata una hiposeñal en T1 y una hiperseñal en T2, sugestivas de un edema medular óseo, en el contexto de una DSR (Fig. 1). Por tanto, se diagnostica el problema de una DSR de la cadera y se inicia el tratamiento con descarga de la articulación, calcitonina intranasal (200 U/día) e indometazina. La paciente evoluciona satisfactoriamente, encontrándose asintomática al mes y medio, por lo que es dada de alta. En la radiografía de control no se evidencian signos de evolución hacia una osteonecrosis.

No vamos a entrar en la discusión de si es más correcto referirse a DSR de la cadera, a osteoporosis transitoria de la cadera, a síndrome de edema transitorio de la médula ósea o incluso si se

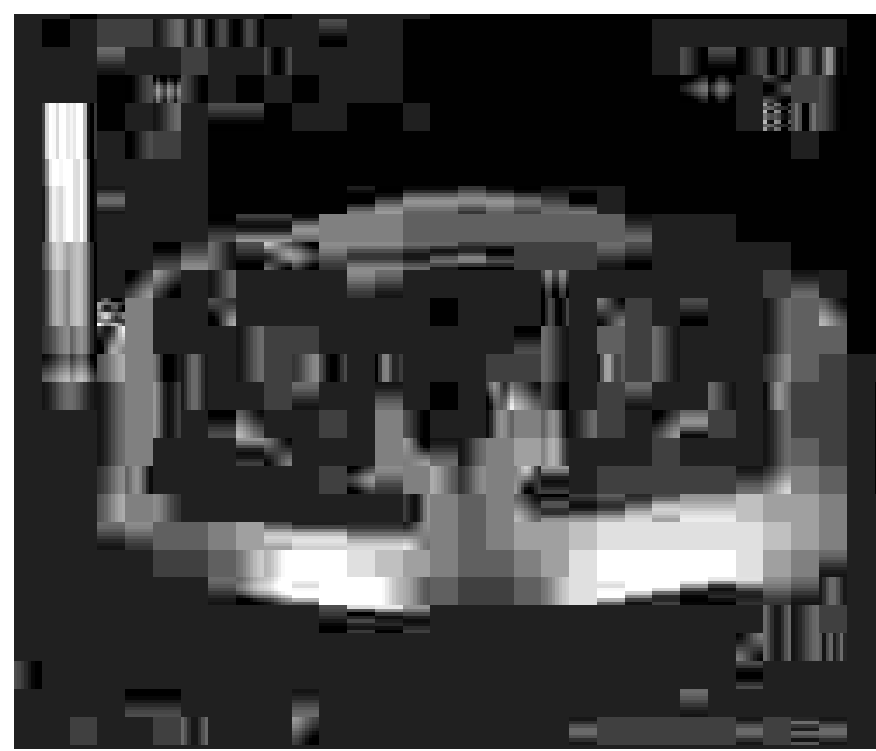

Fig. 1. Distrofia simpático refleja de la cadera izquierda.

trata de una fase inicial de la osteonecrosis, por su falta de interés práctico en esta caso, y por la dificultad de establecer la frontera entre las tres primeras acepciones.

La DSR secundaria al embarazo es un claro ejemplo del polimorfismo clínico de esta entidad. Su prevalencia es variable y difícil de evaluar. Es más frecuente en primíparas, alrededor de los 30 años y, a veces, con sobrepeso. Se presenta en el último tercio del embarazo y, más raramente, en el post-parto, como en el caso expuesto. Suele afectar la cadera, especialmente la izquierda, probablemente por la presentación cefálica del feto que pudiera favorecer microtraumatismos repetidos en dicha cadera. La patogenia de esta DSR no está clara y se involucran diversos factores: los microtraumatismos ya mencionados, la presencia de microfracturas trabeculares, las alteraciones circulatorias propias del embarazo (estasis venoso de la cava inferior), la compresión del simpático pelviano por el útero grávido, las alteraciones lipídicas y una posible disfunción psicológica del embarazo. Clínicamente suele cursar con dolor inguinal, irradiado por la parte anterior del muslo, de aparición súbita o progresiva, de ritmo mecánico, acompañado de impotencia funcional. No obstante, a veces hay formas que pasan desapercibidas, con leve dolor 
pelvi-crural al final del embarazo, y que curan de forma espontánea. El estudio analítico es habitualmente normal y, en ocasiones, pueden observarse alteraciones propias del último tercio del embarazo. La radiografía al inicio es normal, pero entre 1-2 meses se observa una desmineralización habitualmente homogénea, a diferencia de las localizaciones periféricas de la DSR, sin encontrar alteración de la interlínea articular. La gammagrafía muestra, de manera precoz, una hipercaptación del isótopo en el cótilo y en el fémur. La RMN es una exploración importante para el diagnóstico rápido de la afección; suele detectar una hiposeñal en T1 y una hiperseñal en T2, a diferencia de la osteonecrosis que cursa con una hiposeñal en T1 y con una hiposeñal en T2. El diagnóstico se establece con arreglo a los criterios propuestos por Doury. Es conveniente establecer diagnóstico diferencial con una osteonecrosis de cadera, con las formas parciales de la DSR y, en menor medida, con una cruralgia, con patología traumática, con coxitis inflamatorias, con coxitis infecciosas, con tumores de esta zona, con una disfunción del post-parto de la sínfisis del pubis, con patología ginecológica y con patología digestiva. El tratamiento se basa en la descarga de la articulación, antiinflamatorios, calcitonina y rehabilitación. La evolución habitual es hacia la recuperación, completa y sin secuelas, entre algunas semanas y 1 año. No obstante, se ha descrito algún caso de fractura del cuello femoral. La afectación bilateral es relativamente frecuente, incluso es posible una forma migratoria en la rodilla, el tobillo y/o el pie de la misma extremidad. La recidiva en un próximo embarazo también puede verse, pero la recidiva in situ es excepcional (1-10).

En suma, ante todo dolor inguinal y/o en la parte anterior del muslo en una mujer en el último tramo del embarazo o en el postparto, es necesario descartar la presencia de una DSR de la cadera. De igual manera, creemos que es de suma importancia para su diagnóstico precoz la utilización de la gammagrafía y de la RMN, puesto que en la fase inicial la radiología simple, en muchas ocasiones, es normal. Por tanto, resulta fundamental el diagnóstico y el tratamiento, lo más precoz posible, para conseguir una rápida recuperación del proceso. Por último, es conveniente vigilar una posible evolución hacia una osteonecrosis o hacia una posible fractura del cuello femoral.

\section{Bruscas Izu, J.B. Calatayud Pérez, A. Gracia Galve*, R. Arregui Calvo}

Servicio de Reumatología. Servicio de Neurocirugía. *Servicio de Medicina Preventiva. Hospital MAZ. Zaragoza

1. Bruscas Izu C, Medrano San Ildefonso M, Simon L. El diagnóstico de la distrofia simpático refleja. An Med Interna (Madrid) 1999; 16: 308-14.

2. Bonnefoy-Cudraz M, Mazieres B, Fauchier C, et al. Algodystrophie et grossesse. Rev Rhum Mal Osteoartic 1982; 49: 771-4.

3. Bruscas Izu C, Jiménez Aznárez A, Sancho Serrano MA et al. Algodistrofia de cadera y embarazo. An Med Interna (Madrid) 1992; 9: 134-136.

4. Malghem J, Vande Berg B, Lecouvet F, et al. Algodystrophie de la hanche et autres lésions transitoires de la tête fémorale. Rev Rhum Mal Osteartic 1999; 66: 31-40.

5. Bruscas C, Barjon MC, Simon L. Aportación de las exploraciones isotópicas al conocimiento de la algodistrofia. Rev Esp Reumatol 1992: 19: 138-41.

6. Calvo-Crespo E, Alvarez-Galovich L, Fernández-Yruegas Moro D, et al. Síndrome de edema transitorio de la médula ósea (osteoporosis transitoria). Estudio evolutivo por resonancia magnética. Rev Ortop Traumatol 1998; 42: 174-81.

7. Doury P, Dirheimer Y, Pattin S. Algodystrophy: diagnosis and therapy of a frequent disease of the locomotor apparatus. Part 5, Springler-Verlag, Berlin, 1981: 102-3.

8. Sarrat P, Bouscarle P, Felix T, et al. Etude comparative de la scintigraphie, de la scanographie et de l'IRM dans le diagnostic de l'ostéonécrose de hanche et de l'algodystrophie. J Radiol 1985; 66: 779-87.

9. Montella BJ, Nunley JA, Urbaniak JR. Osteonecrosis of the femoral head associated with pregnancy: A preliminary report. J Bone Surg (Am) 1999; 81: 790-8

10. Brocq O, Simon E, Bongain A, et al. Fracture du col fémoral compliquant une algodystrophie au cours de la grossesse. Presse Medicale 1999; 28: 5-6.

\section{Neutropenia severa inducida por ticlopidina. Presentación de dos casos y revisión de la literatura}

\section{Sr. Director:}

La ticlopidina es un antiagregante plaquetario que ha demostrado ser tan eficaz como la aspirina (AAS) en la prevención secundaria del accidente cerebrovascular (ACV) (1-2), enfermedad vascular periférica (3) y cardiopatía isquémica (4), pero puede causar trastornos hematológicos graves. Presentamos 2 casos de netropenia severa asociada al tratamiento con ticlopidina.

Caso 1: mujer de 75 años hipertensa, que presentó varios episodios de accidente isquémico transitorio (AIT) a pesar del empleo de AAS, cambiándose a ticlopidina. Un mes después ingresó por presentar en las últimas 48 horas fiebre y disminución del nivel de conciencia. En la exploración destacaba situación de coma, cianósis, con hipoventilación global, abdomen sin signos de irritación peritoneal. No presentaba focalidad neurológica. Hemograma: leucocitos $1.000 / \mathrm{mm}^{3}$ con $32,4 \%$ neutrófilos (324 neutrófilos $/ \mathrm{mm}^{3}$ ) y $57,4 \%$ linfocitos, $\mathrm{Hgb} 14,4 \mathrm{~g} / \mathrm{dl}$, plaquetas $128.000 / \mathrm{mm}^{3}$. Glucosa $236 \mathrm{mg} / \mathrm{dl}$, creatinina 1,4 , úrea 88 $\mathrm{mg} / \mathrm{dl}$, sodio $138 \mathrm{mEq} / \mathrm{l}$, potasio 4,41 mEq/l. $\mathrm{pH} \mathrm{7,48,} \mathrm{pO}_{2} 71$ $\mathrm{mmHg}, \mathrm{pCO}_{2} 23 \mathrm{mmHg}$. Orina: hematuria y leucocituria. TAC craneal, EKG, radiografía de tórax, punción lumbar y ecocardiograma normales. Ante la sospecha de sepsis de origen urinario se pautó tratamiento con ceftriaxona y se suspendió la ticlopidina, a pesar de lo cual la paciente evolucionó desfavorablemente, presentando anuria, hipotensión y descenso de la cifra de neutrófilos hasta cero falleciendo en 24 horas. No se autorizó necropsia.

Caso 2: varón de 70 años con antecedentes de HTA y cardiopatía isquémica. Cuatro meses antes del ingreso consultó por AIT vertebrobasilar indicándose tratamiento con AAS $600 \mathrm{mg} / \mathrm{día}$. Dos meses antes del ingreso presentó nuevo episodio de AIT sustituyéndose la AAS por ticlopidina. Un día antes del ingreso comenzó con fiebre, mal estado general, tos y expectoración amarillenta. En la exploración física no se objetivaron hallazgos patológicos. Hemograma: leucocitos $750 / \mathrm{mm}^{3}$ con $15 \%$ de neutrófilos $\left(100\right.$ neutrófilos $\left./ \mathrm{mm}^{3}\right)$ y $70 \%$ linfocitos, $\mathrm{Hgb} 12,7 \mathrm{~g} / \mathrm{dl}$, plaquetas 296.000/mm $\mathrm{m}^{3}$. Bioquímica con perfil hepático dentro de la normalidad. Estudio de coagulación, gases sanguíneos y sedimento de orina normales. Baciloscopias y cultivos de esputo negativos. En los hemocultivos creció Escherichia coli. Radiografía de tórax, EKG, ecocardiografía transtorácica y ecografia abdominal dentro de la normalidad.

Se suspendió la ticlopidina y se instauró tratamiento con imipenem y G-CSF (30 millones de unidades). El décimo día se suspendió el G-CSF presentando 4.440 leucocitos $/ \mathrm{mm}^{3}$ con 2.200 neutrófilos $/ \mathrm{mm}^{3}$. La evolución fue satisfactoria con recuperación clínica y hematológica completa.

La incidencia de neutropenia severa (leucocitos inferiores a $1.200 / \mathrm{mm}^{3}$ ) en los estudios con ticlopidina para prevención de AVC ha sido de $0,8-0,9 \%$ (1 2). La aparición de neutropenia suele aparecer entre uno y tres meses después de iniciar el tratamiento y habitualmente revierte a 2-3 semanas de discontinuarla (2). Sin embargo se han descrito casos de neutropenia severa tanto a los 15 días del inicio de la ticlopidina (5) como después de 3 semanas de discontinuarla (6). En nuestro primer caso, a pesar de suspender la ticlopidina, y administrar antibioterapia, las cifras de neutrófilos descendieron hasta cero, con sepsis posterior y fallecimiento del paciente. En la literatura son escasos los casos comunicados de neutropenia severa por ticlopidina con resultado de muerte (7-10), siendo este el tercero comunicado en nuestro pais (búsqueda realizada en BIOSIS, EMBASE y MEDLINE en los años 1989-1999 usando como palabras claves ticlopidine, adverses effects, neutropenia, fatal outcome $y$ death). Hasta 1994, en una búsqueda realizada en una base de datos mundial de comunicación de efectos adversos (Roche Global Pharmacoepidemiology), se encontraron un total de 645 casos de 